\title{
Remarks on Noncommutative Field Theories
}

\author{
M. Gomes \\ Instituto de Física, Universidade de São Paulo, Caixa Postal 66318, 05315-970, São Paulo, SP, Brazil
}

Received on 5 January, 2004 Some of the motivations and basic properties of noncommutative field models are presented. I also comment
on recent developments in the understanding of these peculiar and intriguing theories.

A basic problem, which has accompanied the development of quantum field theory since its inception to our days, concerns the ultraviolet divergences of the perturbative calculations. Already in the thirties, it was suggested that a possible solution for this problem could come from a detailed study of the short distance structure of the space-time. The introduction of a momentum space cutoff that would exclude the ultraviolet region from the calculations seemed inevitable, but the implementation of this mechanism was not and still is not an easy task. So it comes with no surprise that after the success of the renormalization program for quantum electrodynamics in the second half of the forties, this idea was practically abandoned. The renormalization program met a precise formulation in the works of BPHZ and BRST, in the late sixties and beginning of the seventies. It is fair to say that, with the exception of the gravitation, there is a satisfactory understanding of the basic interactions, at least as far as renormalization is concerned.

In the analysis of physical systems sometimes it is not convenient to use a more complete theory but instead an effective model where irrelevant degrees of freedom are eliminated or, more technically, are summed over. An example of such practical attitude comes from the studies of the hydrogen atom: to determine the energy levels it is not necessary to know that the proton is made of quarks; however to obtain the hyperfine structure of the levels, one should take into account that the proton is a spin $1 / 2$ fermion. Similarly, in the study of the low-energy weak decays of elementary particles, the Fermi current-current model can be used instead of the $S U(2) \times U(1)$ electroweak theory.

It is a generalized idea that all useful theories are effective models, some resulting from simplifications in more precise theories, whereas other originate from our incomplete knowledge and perhaps should be obtained from more complete theories whose precise formulations are not yet known. Effective theories (for a recent review see [1]) are supposed to furnish good approximations for energies below to some typical energy scale. As the energy scale is increased new effects are discovered, new stages of knowledge are reached and with luck the old theory is replaced by a new one that explains the new phenomena and so has a larger validity range. We do not know how this process ends. However, even with our limited knowledge we may wonder what would happen at very high energies, if energies of the same order as the Planck's mass were attained. It has been heuristically argued [2] that at such energy scale all measurements of a particle position would loose any operational meaning, due to the formation of a strong gravitational field (a blackhole). Indeed, to measure a coordinate, $x$, let us say, with an accuracy $a$ leads to an indeterminacy in the $x$ component of the momentum of the order of $1 / a$. An energy of the same order may then be transmitted to the system and the corresponding energy-momentum tensor generates a gravitational field which is a solution of the Einstein's equation

$$
R_{\mu \nu}-\frac{1}{2} R g_{\mu \nu}=8 \pi T_{\mu \nu}
$$

The more precise the measurement of the coordinates the bigger the gravitational field coming from the referred measurement. Whenever this field is so strong as to prevent light or other signs to come out from the region under observation, no operational meaning can be given to the idea of strict localization. In this case, the field interactions are not local and infinite renormalizations which are associated with strict locality would not appear. To investigate if such possibility is indeed realized we can postulate the following commutation relations among the coordinates $q^{\mu}$

$$
\left[q^{\mu}, q^{\nu}\right]=i \theta^{\mu \nu}
$$

where for simplicity the $\theta^{\mu \nu}$ matrix is assumed to be constant. Functions of the coordinates no longer commute and therefore it is necessary to furnish rules prescribing as they should be multiplied. Each consistent set of rules defines a quantization scheme. In the Weyl quantization scheme a given classical function $f(x)$ of the commuting coordinates $x_{i}$ and the operator $\hat{f}(q)$ are related by

$$
\hat{f}(q)=\int \frac{d^{n} k}{(2 \pi)^{n}} T(k) \tilde{f}(k),
$$

where $\tilde{f}(k)=\int d^{n} x \mathrm{e}^{i k_{\mu} x^{\mu}} f(x)$ is the Fourier transform of $f(x)$ and the operator $T(k)=\mathrm{e}^{-i k \cdot q}$ satisfies

$$
\begin{array}{ll}
\text { 1. } & T^{\dagger}(k)=T(-k) \\
\text { 2. } & T(k) T\left(k^{\prime}\right)=T\left(k+k^{\prime}\right) \mathrm{e}^{-\frac{i}{2} k_{\mu} k_{\nu}^{\prime} \theta^{\mu \nu}}
\end{array}
$$


A very simple example of the use of this ordering is the one associated with the product of two coordinates. In that case, we have

$$
f(x)=x_{1} x_{2} \quad \Rightarrow \quad \hat{f}(q)=\frac{1}{2}\left(q_{1} q_{2}+q_{2} q_{1}\right),
$$

so that the Weyl prescription implies in the symmetrization of the quantum operators.

The inverse of (3) is most easily obtained after the introduction of the trace $\mathrm{Tr}$, a cyclic operation which maps products of operators in classical functions. We choose it to be normalized such that

$$
\operatorname{Tr}[T(k)]=(2 \pi)^{n} \delta(k) .
$$

We then have $\operatorname{Tr} \hat{f}=\tilde{f}(0)=\int d^{n} x f(x), \operatorname{Tr}\left[\hat{f} T^{\dagger}(k)\right]=$ $\tilde{f}(k)$ and therefore

$$
f(x)=\int \frac{d^{n} k}{(2 \pi)^{n}} \mathrm{e}^{-i k x} \operatorname{Tr}\left[\hat{f} T^{\dagger}(k)\right]
$$

We can now determine the classical function associated to a product of two operators $\hat{f}(q)$ e $\hat{g}(q)$, the so called Moyal product of $f$ and $g$ :

$$
\begin{aligned}
& f(x) * g(x)=\int \frac{d^{n} k}{(2 \pi)^{n}} \mathrm{e}^{-i k x} \operatorname{Tr}\left[\hat{f} \hat{g} T^{\dagger}(k)\right] \\
= & \int \frac{d k}{(2 \pi)^{n}} \int \frac{d k_{1}}{(2 \pi)^{n}} \int \frac{d k_{2}}{(2 \pi)^{n}} \mathrm{e}^{-i k x-\frac{i}{2} k_{1}^{\mu} \Theta_{\mu \nu} k_{2}^{\nu}} \\
\times & \operatorname{Tr}\left[T\left(k_{1}+k_{2}\right) T^{\dagger}(k)\right] \tilde{f}\left(k_{1}\right) \tilde{g}\left(k_{2}\right) \\
= & \int \frac{d k_{1}}{(2 \pi)^{n}} \frac{d k_{2}}{(2 \pi)^{n}} \tilde{\mathrm{e}}^{-i\left(k_{1}+k_{2}\right) x-\frac{i}{2} k_{1}^{\mu} \Theta_{\mu \nu} k_{2}^{\nu}} \\
\times & \tilde{f}\left(k_{1}\right) \tilde{g}\left(k_{2}\right)=\lim _{y \rightarrow x} \mathrm{e}^{\frac{i}{2} \Theta^{\mu \nu} \frac{\partial}{\partial y^{\mu}} \frac{\partial}{\partial x^{\nu}}} f(y) g(x) .
\end{aligned}
$$

In particular,

$$
\begin{aligned}
& {\left[x^{1}, x^{2}\right]_{*} \equiv x^{1} * x^{2}-x^{2} * x^{1}=i \theta^{12}} \\
& x^{1} * x^{2}+x^{2} * x^{1}=2 x^{1} x^{2} .
\end{aligned}
$$

We can also verify that

$$
\begin{aligned}
& f(x) * g(x) * h(x)=\mathrm{e}^{\frac{i}{2} \Theta^{\mu \nu}\left[\frac{\partial^{2}}{\partial x^{\mu} \partial y^{\nu}}+\frac{\partial^{2}}{\partial x^{\mu} \partial z^{\nu}}+\frac{\partial^{2}}{\partial y^{\mu} \partial z^{\nu}}\right]} \\
& \quad \times\left. f(x) g(y) h(z)\right|_{y=z=x}
\end{aligned}
$$

from which a generalization for the product of $n$ operators is straightforward.

During the recent years noncommutative field theories, obtained by replacing the ordinary pointwise product of fields by the Moyal product, have been under intensive study (see [3] for reviews). This interest was greatly motivated by the fact that noncommutative theories emerge as the low energy limit of the open superstring in the presence of an antisymmetric background field [4]. Nevertheless, nowadays noncommutative theories are interesting in their own right. In the sequel we are going to describe some of their intriguing properties.

From the above expressions it is clear that one should proceed carefully when computing time ordered functions of a Moyal product of fields as exponentials containing time derivatives do not commute with the time ordering operation. By forgetting momentarily this problem and writing the mentioned exponentials on the left of the time ordering symbol, one is led to the calculation of graphs topologically similar to those occurring in the commutative situation but with additional phases at the vertices. In momentum space, such phases may turn convergent some of the integrals because they provide strong oscillations in the integrands. However, in certain circumstances some of the phases cancel producing integrals as divergent as in the commutative cases. Whenever the phases are canceled out, the contribution is called planar; otherwise it is said to be nonplanar.

There are two points of concern when considering models defined by replacing the ordinary (pointwise) product of fields by the Moyal one. Firstly, if the noncommutativity involves the time coordinate, i.e. if $\theta_{0 i} \neq 0$ then unitarity and causality are in jeopardy [5]. Let us illustrate this by considering the lowest order correction to the two point function in a theory of a self-interacting scalar field [6] whose action is given by

$$
S_{\text {int }}=\int d^{4} x\left[\frac{1}{2} \partial_{\mu} \phi \partial^{\mu} \phi+\frac{m}{2} \phi^{2}+g \phi * \phi * * \phi\right] .
$$

The corresponding amplitude is proportional to (see Fig. 1)

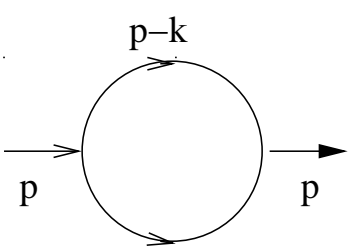

$\mathrm{k}$

Figure 1. Lowest order contribution to the self-energy of the $\phi$ field in the model (12).

$$
\int \frac{d^{4} k}{(2 \pi)^{4}} \frac{1+\cos (p \wedge k)}{\left[(p-k)^{2}-m^{2}+i \epsilon\right]\left(k^{2}-m^{2}+i \epsilon\right)} .
$$

where $p \wedge k=\theta^{\mu \nu} p_{\mu} k_{\nu}$. In coordinate space, redefining $\theta_{\mu \nu} \rightarrow 2 \theta_{\mu \nu}$, we have

$$
\Delta_{F}(x) \Delta_{F}(x)+\Delta_{F}(x) * \Delta_{F}(x) .
$$

It is clear that the first term in the above expression respects unitarity:

$$
\Delta_{F}^{2}+\overline{\Delta_{F}^{2}}=\Delta_{-}^{2}+\Delta_{+}^{2}
$$

where $\Delta_{+}\left(\Delta_{-}\right)$is the positive (negative) frequency part of the Pauli-Jordan commutator function, $\Delta$, and the bar upstairs indicates complex conjugation. The second term, on the other hand, furnishes 


$$
\begin{array}{ll}
\Delta_{F} & (x) * \Delta_{F}(x)+\overline{\Delta_{F}(x) * \Delta_{F}(x)} \\
=\quad & \Delta_{+} * \Delta_{+}+\Delta_{-} * \Delta_{-} \\
& +\Delta_{r e t} * \Delta_{a v}+\Delta_{a v} * \Delta_{r e t},
\end{array}
$$

where $\Delta_{a v}$ and $\Delta_{r e t}$ designate the advanced and retarded Green functions of the free scalar field theory. The breakdown of unitarity is a direct consequence of the nonvanishing of the last two terms. However, if the noncommutativity does not affect the time, i.e., if $\theta_{0 i}=0\left(h \equiv h\left(x^{0}\right)\right.$ represents the Heaviside step function)

$$
\Delta_{\text {ret }} * \Delta_{a v} \approx[h(1-h)] \Delta * \Delta=0 .
$$

It has been argued that the unitarity problem can be overcome by firstly calculating the Moyal product and then applying the time ordering prescription $[6,7]$. For the case of the Fig. 1, this means that the corresponding amplitude now involves objects of the type

$$
h \Delta_{+} * \Delta_{+}+(1-h) \Delta_{-} * \Delta_{-} .
$$

But,

$$
\begin{aligned}
& h \Delta_{+} * \Delta_{+}+(1-h) \Delta_{-} * \Delta_{-}+h \overline{\Delta_{+} * \Delta_{+}} \\
& +(1-h) \overline{\Delta_{-} * \Delta_{-}}=\Delta_{+} * \Delta_{+}+\Delta_{-} * \Delta_{-}
\end{aligned}
$$

and thus unitarity holds since the last expression also arises in the product of two tree graphs. The procedure just described leads to much more complicated and laborious computations than in the ordinary Feynman graph calculations. Further studies are needed since recent developments indicate that in noncommutative gauge theories unitarity may still be broken insofar as the Ward identities which enforce the cancellation of unphysical degrees of freedom are not satisfied [8].

Another problem characteristic of noncommutative theories is the ultraviolet-infrared (UV-IR) mixing. The nomenclature indicates a mixture of scales typical of nonlocal models. We recall that in the usual situation of commutative theories, the ultraviolet behavior of Feynman integrals is unrelated to the infrared one. By contrast, in the noncommutative setting, the nonplanar part of the integrals contain trigonometric factors which imply in the ultraviolet finiteness but lead to results that are singular at small momenta. An illustrative example is provided by the tadpole graph of the $\varphi^{4}$ model, depicted in the Fig. 2. The nonplanar part of that graph is given by

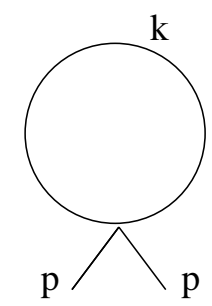

Figure 2. A tadpole graph of the noncommutative $\varphi^{4}$ model.

$$
\int \frac{d^{4} k}{(2 \pi)^{4}} \frac{\cos (k \wedge p)}{k^{2}-m^{2}}
$$

and, thanks the oscillations of the cosine, the integral turns out to be convergent. However, for small momenta it behaves as $\frac{\zeta}{\tilde{p}^{2}}$ where $\tilde{p}^{\mu} \equiv \theta^{\mu \nu} p_{\nu}$ and $\zeta$ is a numerical constant. This is a troublesome result as multiple insertions of the tadpole graph into a larger diagram produce nonintegrable singularities implicating in the breakdown of the perturbative expansion. Besides, it is clear that the presence of these infrared singularities may also lead to a violation of unitarity [9].

In the usual commutative theories, the existence of infrared divergences signalizes that nonperturbative effects must be incorporated. One may try resummations, rearranging the perturbative series to obtain a better behaved expansion. A difficulty in such procedure is the identification of a perturbative parameter to control the resummed series. Another possibility is to increase the model by adding new degrees of freedom that hopefully cancel the singularities. In this aspect supersymmetry may play a decisive role since, at least in the commutative situation, many cancellations of ultraviolet divergences occur in supersymmetric models. A first concrete example in this direction was given by the noncommutative Wess-Zumino model where renormalizability was proven to all loop orders [10]. Gauge invariance brings some additional complications and no general statement, valid to all orders, exist. Actually, up to one-loop, in the case of supersymmetric $\mathrm{QED}_{4}$, by employing the formalism of components fields, it was shown that the contributions to the gauge field two and three point functions are free of dangerous (i.e., quadratic or linear) infrared divergences [11]. By using powerful superfield techniques, this analysis has been improved $[12,13]$. Indeed, by working in an arbitrary covariant gauge it was proven that the cancellation of the quadratic infrared divergences also hold for extended supersymmetry. For the case of the three point function of the gauge superfield where the infrared divergences are at most linear, the dangerous linear infrared divergences turn out to be a gauge artifact disappearing in a particular gauge.

At first glance one could think that the dangerous effects of the UV-IR mixing should be absent in theories whose commutative counterparts is characterized by cancellations of the quadratic and linear ultraviolet divergences. This is not correct because the noncommutativity modifies the counterterm structure. Examples where such situation occurs are the $(2+1)$ dimensional Gross-Neveu model [14] and the four dimensional linear sigma model [15]. A characteristic feature of these models is the fact that the renormalization of the coupling constant plays a dual role: it eliminates tadpoles and also the leading ultraviolet divergences of some two point functions (the two point function of the auxiliary field in the three-dimensional Gross-Neveu model, the two point function of the pion field in the four dimensional linear sigma model). In the four dimensional linear sigma model it has been proved that spontaneous breakdown may occur for the $U(N)$ but not for the $O(N)$ if $N \neq 2[15,16]$. Even though the Goldstone theorem holds only if the field ordering in the quartic Moyal product is consistent with local 
symmetry. In $2+1$ dimensions the situation concerning the Goldstone theories is more favorable: In a model of complex scalar fields coupled to a combination of quartic and sextuple self-interactions, there is a class of field orderings for which no UV-IR mixing occurs and massless excitations appear. Unlike the four dimensional case this does not require gauge invariant couplings [17].

Recent developments in $2+1$ dimensions unveiled other interesting properties: as it happens in its commutative version, the noncommutative $\mathrm{CP}^{N-1}$ model, with the basic fields belonging to the fundamental representation of the gauge group, turns out to be renormalizable up to the next to leading order of $1 / N$. This is true in spite of the fact that, due to the lack of charge conjugation [18], new UV divergent graphs appear whereas some amplitudes that in the commutative version were divergent gained oscillatory factors and become finite [19]. In contrast, if the basic field belongs to the adjoint representation dangerous infrared singularities are present in the two point function of the gauge field and also in the leading correction to the self energy of the scalar field. These singularities still persist if fermions are minimally coupled to the gauge field but disappear under supersymmetrization.

The existence of the UV-IR mixing implies in a modification of the dispersion relation. For example in the case of the self-interacting scalar theory defined by (12) the usual dispersion relation $p_{0}^{2}=\vec{p}^{2}+m^{2}$ is deformed to $p_{0}^{2}=\vec{p}^{2}+m^{2}+\zeta / \tilde{p}^{2}$. This fact may have relevant implications in astrophysical observations involving particles of very high energy [20].

Another surprising aspect is the appearance of noncommutative solitons in theories which do not allow them if the setting were commutative. In fact, in ordinary scalar theories Derrick's theorem [21] prevents the occurrence of solitons if the spacetime dimension is bigger than two. However, it has been shown that for large values of the noncommutativity parameter solitons exist even in scalar models [22] (see also [23]).

In 1+1 dimensions noncommutative soliton solutions have, of course, the same analytic expression as the commutative ones. A distinctive feature of noncommutativity in these models arises from the fact that the noncommutative solitons lead to the creation of new bound states or even to an infinite tower of bound states; this property has been explicitly verified in the $\phi^{4}$ and sine-Gordon models [24].

We have seen that the introduction of noncommutative spaces in field theory produced an avenue with many interesting new constructions. Nevertheless, if they are theoretical inspirations that could lead to a consistent picture of the world at very small scale is yet to be seen.

\section{Acknowledgments}

This work was partially supported by Fundação de Amparo à Pesquisa do Estado de São Paulo (FAPESP) and Conselho Nacional de Desenvolvimento Científico e Tecnológico (CNPq).

\section{References}

[1] I. Z. Rothstein, "TASI Lectures on Effective Field Theories", hep-ph/0308266.

[2] S. Doplicher, K. Fredenhagen, and J. E. Roberts, Commun. Math. Phys. 172, 187 (1995).

[3] N.A. Nekrasov and M. Douglas, Rev. Mod. Phys. 73, 977 (2002); R. J.Szabo, Phys. Rept. 378, 207 (2003); M. Gomes, "Renormalization in Noncommutative Field Theory" in Proceedings of the XI Jorge André Swieca Summer School, Particles and Fields" G. A. Alves, O. J. P. Éboli, and V. O. Rivelles eds, World Scientific Pub. Co, 2002; H. O. Girotti, "Noncommutative Quantum Field Theories", hep-th/0301237.

[4] N. Seiberg and E. Witten, J. High Energy Phys. 09, 32 (1999).

[5] J. Gomis and T. Mehen, Nucl. Phys. B591, 265 (2000); N. Seiberg, L. Susskind, and N. Toumbas, J. High Energy Phys. 006, 044 (2000).

[6] D. Bahns, S. Doplicher, K. Fredenhagen, and G. Piacitelli, Phys. Lett. B533, 178 (2002); C. Rim and J. H. Yee, Phys. Lett. B574, 111 (2003).

[7] Yi Liao and K. Sibold, Eur. Phys. J. C 25, 479 (2002); S. Denk and M. Schweda, J. High Energy Phys. 0309, 032 (2003).

[8] T. Ohl, R. Rückl, and J. Zeiner, Nucl. Phys. B676, 229 (2003).

[9] Chong-Sun Chu, J. Lukierski and W. Zakrzewski, Nucl. Phys. B632, 219 (2002).

[10] H. O. Girotti, M. Gomes, V. O. Rivelles, and A. J. da Silva, Nucl. Phys. B587, 299 (2000); A. A. Bichl, J. M. Grimstrup, H. Grosse, L. Popp, M. Schweda, and R. Wulkenhaar, J. High Energy Phys. 0010, 046 (2000).

[11] A. Matusis, L. Susskind, and N. Toumbas, J. High Energy Phys. 12, 002 (2000); F. Ruiz Ruiz, Phys. Lett. B502, 274 (2001).

[12] A. A. Bichl, M. Ertl, A. Gerhold, J. M. Grimstrup, H. Grosse, L. Popp, V. Putz, M. Schweda, and R. Wulkkenhaar, "Noncommutative U(1) Super-Yang-Mills Theory: Perturbative Self-Energy Corrections", hep-th/0203141.

[13] A. F. Ferrari, H. O Girotti, M. Gomes, A. Yu. Petrov, A. A. Ribeiro, V. O. Rivelles, and A. J. da Silva, "Superfield Covariant Analysis of the Divergence Structure of Noncommutative $\mathrm{QED}_{4}$ ”, Phys, Rev. D. to appear, hep-th/0309154.

[14] H. O. Girotti, M. Gomes, V. O. Rivelles, and A. J. da Silva, Int. J. Mod. Phys. A17, 1503 (2002).

[15] B. A. Campbell and K. Kaminsky, Nucl. Phys. B581, 240 (2000); Nucl. Phys. B606, 169 (2001).

[16] F. J. Petriello, Nucl. Phys. B601, 169 (2001).

[17] H. O. Girotti, M. Gomes, V. O. Rivelles, and A. J. da Silva, Phys. Rev. D67, 125003 (2003).

[18] M. M. Sheikh-Jabbari, Phys. Rev. Lett. 84, 5265 (2000), hepth/0001167; M. Chaichian, K. Nishijima, and A. Tureanu, Phys. Lett. B568, 146 (2003), hep-th/0209008.

[19] E. A. Asano, M. Gomes, A. G. Rodrigues, and A. J. da Silva, "The $(2+1)$ D Noncommutative $\mathrm{CP}^{N-1}$ Model", Phys. Rev. D. to appear, hep-th/0307114.

[20] G. Amelino-Camelia, L. Doplicher, S. Nam, and Yun-Seok Seo, Phys. Rev. D67, 085008 (2003). 
[21] G. Derrick, J. Math. Phys. 5, 1252 (1964).

[22] R. Gopakumar, S. Minwalla, and A. Strominger, J. High Energy Phys. 0005, 020 (2000).

[23] J. A. Harvey, "Komaba Lectures on Noncommutative So- litons and D-Branes", hep-th/0102076; F. A. Schaposnik, "Noncommutative Solitons and Instantons", hep-th/0310202.

[24] D. V. Vassilevich and A. Yurov, "Space-time Noncommutativity Tends to Create Bound States", hep-th/0311214. 